\title{
Quasi-Linear Algebras and Integrability (the Heisenberg Picture) ${ }^{\star}$
}

Luc VINET ${ }^{\dagger}$ and Alexei ZHEDANOV ${ }^{\ddagger}$

$\dagger$ Université de Montréal PO Box 6128, Station Centre-ville, Montréal QC H3C 3J\%, Canada E-mail: luc.vinet@umontreal.ca

$\ddagger$ Donetsk Institute for Physics and Technology, Donetsk 83114, Ukraine

E-mail: zhedanov@yahoo.com

Received November 16, 2007, in final form January 19, 2008; Published online February 06, 2008

Original article is available at http://www.emis.de/journals/SIGMA/2008/015/

\begin{abstract}
We study Poisson and operator algebras with the "quasi-linear property" from the Heisenberg picture point of view. This means that there exists a set of one-parameter groups yielding an explicit expression of dynamical variables (operators) as functions of "time" $t$. We show that many algebras with nonlinear commutation relations such as the Askey-Wilson, $q$-Dolan-Grady and others satisfy this property. This provides one more (explicit Heisenberg evolution) interpretation of the corresponding integrable systems.
\end{abstract}

Key words: Lie algebras; Poisson algebras; nonlinear algebras; Askey-Wilson algebra; Dolan-Grady relations

2000 Mathematics Subject Classification: 17B63; 17B37; 47L90

\section{Classical version}

Assume that there is a classical Poisson manifold with the Poisson brackets (PB) $\{x, y\}$ defined for all dynamical variables $x, y$ belonging to this manifold. Of course, it is assumed that the PB satisfy the standard conditions:

(i) $\left\{x, \alpha_{1} y+\alpha_{2} z\right\}=\alpha_{1}\{x, y\}+\alpha_{2}\{x, z\}-$ linearity $\left(\alpha_{1}, \alpha_{2}\right.$ are arbitrary constants);

(ii) $\{x, y\}=-\{y, x\}-$ antisymmetricity;

(iii) $\{x, y z\}=z\{x, y\}+y\{x, z\}-$ the Leibnitz rule;

(iv) $\{\{x, y\}, z\}+\{\{y, z\}, x\}+\{\{z, x\}, y\}=0-$ the Jacobi identity.

If one chooses the "Hamiltonian" $H$ (i.e. some dynamical variable belonging to this manifold), then we have the standard Hamiltonian dynamics: all variables $x(t)$ become depending on an additional time variable $t$ and the equation of motion is defined as

$$
\dot{x}(t)=\{x(t), H\} .
$$

Of course, the Hamiltonian $H$ is independent of $t$, because $\dot{H}=\{H, H\}=0$. More generally, a dynamical variable $Q$ is called the integral of motion if $\dot{Q}=0$. Clearly, this is equivalent to the condition $\{Q, H\}=0$.

From (1.1) we have $\ddot{x}=\{\{x, H\}, H\}$ and more generally

$$
\left.\frac{d^{n} x}{d t^{n}}=\{\ldots\{x, H\}, H\}, \ldots H\right\}=\left\{x, H^{(n)}\right\},
$$

${ }^{\star}$ This paper is a contribution to the Proceedings of the Seventh International Conference "Symmetry in Nonlinear Mathematical Physics" (June 24-30, 2007, Kyiv, Ukraine). The full collection is available at http://www.emis.de/journals/SIGMA/symmetry2007.html 
where notation $\left\{x, H^{(n)}\right\}$ means $n$ repeated PB. Using this formula we can write down the formal Taylor expansion of an arbitrary dynamical variable in the form

$$
\begin{aligned}
x(t) & =x(0)+t \dot{x}(0)+\cdots+\left.\frac{t^{n}}{n !} \frac{d^{n} x(t)}{d t^{n}}\right|_{t=0}+\cdots \\
& =x(0)+t\{x(0), H\}+\cdots+\frac{t^{n}}{n !}\left\{x(0), H^{(n)}\right\}+\cdots .
\end{aligned}
$$

Assume now that there are $N$ dynamical variables $x_{1}(0), x_{2}(0), \ldots, x_{N}(0)$ (not depending on $t$ ) with the following property: for every $k=1, \ldots, N$ the $\mathrm{PB}$ of the variable $x_{k}(0)$ with the Hamiltonian $H$ depends linearly on all $x_{i}(0)$ :

$$
\left\{x_{k}(0), H\right\}=\sum_{s=1}^{N} F_{k s}(H) x_{s}(0)+\Phi_{k}(H), \quad k=1,2, \ldots, N,
$$

where $F_{k s}(H), \Phi_{k}(H)$ are some functions of the variable $H$ only. Using rules (i)-(iv) for the PB we have

$$
\left\{\left\{x_{k}(0), H\right\}, H\right\}=\sum_{s=1}^{N} F_{k s}(H)\left\{x_{s}(0), H\right\}=\sum_{s=1}^{N} F_{k s}^{(2)}(H) x_{s}(0)+\Phi_{k}^{(1)}(H),
$$

where

$$
F_{k s}^{(2)}(H)=\sum_{i=1}^{N} F_{k i}(H) F_{i s}(H), \quad \Phi_{k}^{(1)}(H)=\sum_{i=1}^{N} F_{k i}(H) \Phi_{i}(H) .
$$

We see that the double $\mathrm{PB}\left\{\left\{x_{k}(0), H\right\}, H\right\}$ is again linear with respect to $x_{i}(0)$.

In what follows we will use the matrix notation assuming that $F(H)$ is the $N \times N$ matrix with the entries $F_{i k}(H)$ and $\Phi(H)$ is the $N$-dimensional vector with the components $\Phi_{i}(H)$. Then it is seen that $F^{(2)}(H)$ is the square of the matrix $F(H)$ and the vector $\Phi^{(1)}$ is obtained by the applying of the matrix $F(h)$ to the vector $\Phi(H): \Phi^{(1)}(H)=F(H) \Phi(H)$ in accordance with usual conventions in linear algebra.

By induction, we obtain the following formula

$$
\left\{x_{k}(0), H^{(n)}\right\}=\sum_{s=1}^{N} F_{k s}^{(n)}(H) x_{s}(0)+\Phi_{k}^{(n-1)}(H),
$$

where the matrix $F^{(n)}(H)$ means the $n$-th power of the matrix $F_{i k}$ and the vector $\Phi^{(n-1)}(H)$ is

$$
\Phi^{(n-1)}(H)=F^{(n-1)} \Phi(H) .
$$

Now using (1.2) we can write down the formula

$$
x_{k}(t)=\sum_{n=0}^{\infty}\left\{x_{k}(0), H^{(n)}\right\} \frac{t^{n}}{n !} .
$$

By (1.4) we can present the above formula in the form

$$
x_{k}(t)=\sum_{s=1}^{N} E_{k s}(H ; t) x_{s}(0)+G_{k}(H ; t),
$$


where the $N \times N$ matrix $E(H ; t)$ and the $N$-dimensional vector $G(H, t)$ are defined as

$$
E(H ; t)=\exp (t F(H)), \quad G(H ; t)=\left(\int_{\tau=0}^{t} \exp (\tau F(H)) d \tau\right) \Phi(H) .
$$

We used the ordinary definition of the exponential function of the matrix:

$$
\exp (t F)=\sum_{n=0}^{\infty} \frac{t^{n} F^{(n)}}{n !}
$$

and

$$
\int_{\tau=0}^{t} \exp (\tau F) d \tau=\sum_{n=0}^{\infty} \frac{t^{n+1} F^{(n)}}{(n+1) !}
$$

We see from (1.5) that every variable $x_{k}(t)$ is a linear function with respect to initial variables $x_{s}(0)$.

Proposition 1. Assume that there is an analytical matrix function $E(H ; t)$ and an analytical vector function $G(H ; t)$ such that condition (1.5) holds for every $k=1,2, \ldots, N$. Then this condition is equivalent to condition (1.3).

Proof of this proposition is elementary.

So far, we did not concretize the choice of the Hamiltonian $H$. Now we assume that all variables $x_{s}(0), s=1, \ldots, N$ can be chosen as Hamiltonians: if one puts $H=x_{s}(0)$ then we will have linearity property (1.3) with respect to all other variables $x_{i}, i \neq s$. It is naturally to call such algebras the quasi-linear Poison algebras. This means that linearity property holds for all generators $x_{i}$, apart from the Hamiltonian variable $x_{s}$.

Quasi-linear algebras possess a remarkable property: if one chooses any basic variable $x_{j}$, $j=1,2, \ldots, N$ as a Hamiltonian then for time evolution of all other variables $x_{i}, i=1, \ldots, N$, $i \neq j$ we have linear property

$$
x_{i}(t)=\sum_{s=1}^{N}{ }^{\prime} \xi_{i j s}\left(x_{j} ; t\right) x_{s}(0)+\eta_{i j}\left(x_{j} ; t\right), \quad i \neq j,
$$

where the functions $\xi_{i j s}\left(x_{j} ; t\right)$ and $\eta_{i j}\left(x_{j} ; t\right)$ can be explicitly calculated as was shown above. Notation $\sum_{s=1}^{N}$ ' means that we exclude the term with $s=j$ from the sum (note that excluding value of $s$ coincides with the fixed "number" $j$ of the Hamiltonian).

In this case we obtain strong restrictions for the expression of the Poisson brackets $\left\{x_{i}, x_{k}\right\}$. Indeed, from this property we have that for any pair of variables $x_{i}, x_{k}$ one has the relation

$$
\left\{x_{i}, x_{k}\right\}=\sum_{s=1}^{N} F_{i k s}\left(x_{k}\right) x_{s}+\Phi_{i k}\left(x_{k}\right), \quad i, k=1,2, \ldots, N, \quad i \neq k
$$

with some functions $F_{i k s}\left(x_{k}\right)$ and $\Phi_{i k}\left(x_{k}\right)$. These functions cannot be taken arbitrarily. Indeed, we can also write down the similar condition but with reversed order of variables $x_{i}, x_{k}$ :

$$
\left\{x_{k}, x_{i}\right\}=\sum_{s=1}^{N} F_{k i s}\left(x_{i}\right) x_{s}+\Phi_{k i}\left(x_{i}\right), \quad i, k=1,2, \ldots, N, \quad i \neq k .
$$


But $\left\{x_{k}, x_{i}\right\}=-\left\{x_{i}, x_{k}\right\}$, so we have the system of conditions

$$
\sum_{s=1}^{N}{ }^{\prime} F_{k i s}\left(x_{i}\right) x_{s}+\Phi_{k i}\left(x_{i}\right)+\sum_{s=1}^{N}{ }^{\prime} F_{i k s}\left(x_{k}\right) x_{s}+\Phi_{i k}\left(x_{k}\right)=0
$$

which should be valid for any pair $x_{i}, x_{k}, i \neq k$.

Moreover, the Jacobi identity

$$
\left\{\left\{x_{i}, x_{k}\right\}, x_{j}\right\}+\left\{\left\{x_{k}, x_{j}\right\}, x_{i}\right\}+\left\{\left\{x_{j}, x_{i}\right\}, x_{k}\right\}=0
$$

should be valid for any triple $x_{i}, x_{k}, x_{j}$ with distinct values $i, j, k$. In our case the Jacobi identity (1.9) is reduced to a system of functional-differential equations

$$
W_{i j k}+W_{j k i}+W_{k i j}=0
$$

where

$$
W_{i j k}=\left\{\left\{x_{i}, x_{k}\right\}, x_{j}\right\}=\Phi_{i k}^{\prime}\left(x_{k}\right)\left\{x_{k}, x_{j}\right\}+\sum_{s=1}^{N^{\prime}}\left(F_{i k s}\left(x_{k}\right)\left\{x_{s}, x_{j}\right\}+F_{i k s}^{\prime}\left(x_{k}\right) x_{s}\left\{x_{k}, x_{j}\right\}\right),
$$

where $F^{\prime}(x)$ means the derivative of the function $F(x)$.

Conditions (1.8) and (1.10) can be considered as a system of nontrivial functional-differential equations for unknown functions $F_{i k s}\left(x_{k}\right)$ and $\Phi_{i k}\left(x_{k}\right)$.

Of course, there is a trivial solution of these conditions when all functions are constants, i.e. $F_{i k s}\left(x_{k}\right)$ and $\Phi_{i k}\left(x_{k}\right)$ do not depend on their arguments $x_{k}$. If, additionally, $\Phi_{i k} \equiv 0$ then we obtain well-known Lie-Poisson algebras with commutation relations

$$
\left\{x_{i}, x_{k}\right\}=\sum_{s=1}^{N} c_{i k s} x_{s}
$$

with the structure constants $c_{i k s}$ satisfying standard restrictions following from (1.8) and (1.10) (see, e.g. [10]).

Consider the most general finite-dimensional Poisson algebras with nonlinear Poison brackets

$$
\left\{x_{i}, x_{k}\right\}=h_{i k}\left(x_{1}, \ldots, x_{N}\right)
$$

where $h_{i k}\left(x_{1}, \ldots, x_{N}\right)$ are smooth functions of $N$ variables $x_{1}, \ldots, x_{N}$. For general theory connected with these algebras see e.g. [10]. In order for the variables $x_{i}, x_{k}$ to form a Poisson algebra, the functions $h_{i k}$ should satisfy some strong restrictions. In particular, in [6] necessary and sufficient conditions were obtained in case when $h_{i k}\left(x_{1}, \ldots, x_{N}\right)$ are quadratic functions. We will provide several examples when Poisson algebras of type (1.11) satisfy conditions (1.8) and (1.10) and hence are quasi-linear. General classification of all Poisson algebras with such property is an interesting open problem.

Note that in [10] the so-called semi-linear Poisson algebras (as well as their operator analogues) were considered. Such algebras are in general nonlinear but they have linearity property with respect to some prescribed generators. However the semi-linear algebras introduced in [10] do not possess, in general, the property (1.7) and hence they are not quasi-linear algebras in our sense. 


\section{2 "Quantum" (operator) version}

Now assume that $X_{k}, k=1,2, \ldots$ are operators which act on some linear space (either finite or infinite-dimensional).

The time dynamics is defined by the Heisenberg equations:

$$
\dot{B}=[H, B],
$$

where $H$ is an operator called the Hamiltonian and $[H, B] \equiv H B-B H$ is the commutator.

As in the previous section, assume that there exist $N$ operators $X_{k}$ and a Hamiltonian $H$ such that the conditions

$$
\left[H, X_{k}\right]=\sum_{s=1}^{N} F_{k s}(H) X_{s}+\Phi_{k}(H),
$$

where $F_{k s}(H), \Phi_{k}(H)$ are some functions depending only on the Hamiltonian $H$.

Introduce the $n$-th repeated commutator

$$
\left[H,[H, \ldots,[H, B] \ldots]=\operatorname{ad}_{H}^{n} B\right.
$$

As in the previous section we have

$$
\operatorname{ad}_{H}^{n} X_{k}=\sum_{s=1}^{N} F_{k s}^{(n)}(H) X_{s}+\Phi_{k}^{(n-1)}(H),
$$

where the matrix $F^{(n)}(H)$ means the $n$-th power of the matrix $F_{i k}$ and the vector $\Phi^{(n-1)}(H)$ is

$$
\Phi^{(n-1)}(H)=F^{(n-1)} \Phi(H) .
$$

The Heisenberg time evolution is described by the one-parametric group in a standard manner

$$
X_{k}(t)=\exp (t H) X_{k} \exp (-t H)=X_{k}+t\left[H, X_{k}\right]+\cdots+\frac{t^{n}}{n !} \operatorname{ad}_{H}^{n} X_{k}+\cdots .
$$

Note that in our approach the time variable $t$ can be an arbitrary complex parameter and the "Hamiltonian" $H$ need not be a Hermitian operator. In physical applications it is usually assumed that $H$ is a Hermitian operator and we need to change $t \rightarrow i t$ in order to obtain the usual Heisenberg picture where the time $t$ is a real parameter.

Again, as in classical case we obtain that $X_{k}(t)$ is a linear combination of initial operators $X_{s}$ :

$$
X_{k}(t)=\sum_{s=1}^{N} E_{k s}(H ; t) X_{s}+G_{k}(H ; t),
$$

where the $N \times N$ operator-valued matrix $E(H ; t)$ and the $N$-dimensional operator-valued vector $G(H, t)$ are defined by the same formulas (1.6).

Note that if the operators $X_{s}$ form a Lie algebra:

$$
\left[X_{i}, X_{k}\right]=\sum_{s=1}^{N} g_{i k}^{s} X_{s}
$$

where $g_{i k}^{s}$ are the structure constants, then any operator $X_{j}=H$ taken as a Hamiltonian, satisfies conditions (2.1) with $F_{k s}$ the constants (not depending on $H$ ) and $\Phi_{k}=0$, hence we 
have formula (2.2) with $G_{k}=0$ which is a standard action of the one-parameter Lie group corresponding to the generator $H=X_{j}$ :

$$
\exp \left(t X_{j}\right) X_{k} \exp \left(-t X_{j}\right)=\sum_{s=1}^{N} E_{k s}(t) X_{s}
$$

Of course, in the case of the Lie algebra we can construct full Lie group due to linearity property ( $g_{i k}^{s}$ are constants). However, if $F_{k s}(H)$ depend on an operator $H$ then we see that only a set of one-parameter groups exists with the linearity property (2.2). Our next problem therefore, will be how to construct algebras satisfying the property (2.1) for different possible choices of the operator $H$.

The simplest possibility is the same as in the previous section: we demand that any operator $X_{j}$ can be taken as a Hamiltonian $H=X_{j}, j=1,2, \ldots, N$. We call such algebras the quasi-linear operator algebras. Condition (2.1) is replaced with a system of conditions

$$
\left[X_{j}, X_{k}\right]=\sum_{s=1}^{N} F_{j k s}\left(X_{j}\right) X_{s}+\Phi_{j k}\left(X_{j}\right), \quad k, j=1,2 \ldots, N, \quad k \neq j .
$$

Then for all possible choices of the Hamiltonian $H$ the time evolution (the Heisenberg picture) has the linearity property with respect to all operators apart from the Hamiltonian:

$$
X_{k}^{(j)}(t)=\exp \left(t X_{j}\right) X_{k} \exp \left(-t X_{j}\right)=\sum_{s=1}^{N}{ }^{\prime} E_{j k s}\left(X_{j} ; t\right) X_{s}+G_{j k}\left(X_{j} ; t\right),
$$

where the functions $E_{j k s}\left(X_{j} ; t\right)$ and $G_{j k}\left(X_{j} ; t\right)$ are easily calculated from the functions $F_{j k s}\left(X_{j}\right)$ and $\Phi_{j k}\left(X_{j}\right)$.

Of course, compatibility analysis of conditions (2.4) as well as checking of the Jacobi identity

$$
\left[\left[X_{i}, X_{k}\right], X_{j}\right]+\left[\left[X_{k}, X_{j}\right], X_{i}\right]+\left[\left[X_{j}, X_{i}\right], X_{k}\right]=0, \quad i, j, k=1,2, \ldots, N
$$

is rather a nontrivial problem even for the case when all operators $X_{k}$ are finite-dimensional. Instead, we present concrete examples of the operator algebras possessing quasi-linear property.

However first we need to generalize our scheme to include the so-called "extension" operators.

\section{Quasi-linear algebras with extension}

Assume that we have a set of $N$ so-called "basic" operators $X_{1}, X_{2}, \ldots X_{N}$ and for any operator $X_{i}, i=1,2, \ldots, N$ we have also "a tower" of extension, i.e. a set of $M_{i} \geq 0$ operators $Y_{k}^{(i)}$, $k=1,2, \ldots, M_{i}$, such that we have the commutation relations

$$
\begin{aligned}
& {\left[X_{i}, X_{k}\right]=\sum_{s=1}^{N}{ }^{\prime} F_{i k s}\left(X_{i}\right) X_{s}+\sum_{s=1}^{M_{i}} G_{i k s}\left(X_{i}\right) Y_{s}^{(i)}+\Phi_{i k}\left(X_{i}\right),} \\
& i, k=1,2, \ldots, N, \quad i \neq k
\end{aligned}
$$

and

$$
\begin{aligned}
& {\left[X_{i}, Y_{k}^{(i)}\right]=\sum_{s=1}^{N} U_{i k s}\left(X_{i}\right) X_{s}+\sum_{s=1}^{M_{i}} V_{i k s}\left(X_{i}\right) Y_{s}^{(i)}+W_{i k}\left(X_{i}\right),} \\
& i=1,2, \ldots, N, \quad k=1,2, \ldots, M_{i}
\end{aligned}
$$


with some functions $F_{i k s}(x), \ldots, W_{i k}(x)$. In what follows we will assume that all these functions are polynomials.

Thus the extension operators $Y_{k}^{(i)}$ enter the linear combinations in commutators (3.1), (3.2) but only operators $X_{i}$ can be chosen as "Hamiltonians". In general, commutation relations between operators $Y_{k}^{(i)}$ are not defined as well as the commutation relations between the Hamiltonian $X_{i}$ and operators $Y_{k}^{(j)}$ from another "tower" (i.e. when $j \neq i$ ).

From (3.1), (3.2) it follows immediately that repeated commutators with the "Hamiltonians" $X_{i}$ have the similar structure

$$
\operatorname{ad}_{X_{i}}^{n} X_{k}=\sum_{s=1}^{N}{ }^{\prime} F_{i k s}^{(n)}\left(X_{i}\right) X_{s}+\sum_{s=1}^{M_{i}} G_{i k s}^{(n)}\left(X_{i}\right) Y_{s}^{(i)}+\Phi_{i k}^{(n)}\left(X_{i}\right), \quad i, k=1,2, \ldots, N
$$

and

$$
\operatorname{ad}_{X_{i}}^{n} Y_{k}^{(i)}=\sum_{s=1}^{N}{ }^{\prime} U_{i k s}^{(n)}\left(X_{i}\right) X_{s}+\sum_{s=1}^{M_{i}} V_{i k s}^{(n)}\left(X_{i}\right) Y_{s}^{(i)}+W_{i k}^{(n)}\left(X_{i}\right)
$$

with polynomials $F_{i k s}^{(n)}(x), \ldots, W_{i k}^{(n)}(x)$ which can be obtained from $F_{i k s}(x), \ldots, W_{i k}(x)$ by obvious explicit procedures.

Hence we have an explicit Heisenberg evolution picture:

$$
e^{t X_{i}} X_{k} e^{-t X_{i}}=\sum_{s=1}^{N} \tilde{F}_{i k s}\left(X_{i} ; t\right) X_{s}+\sum_{s=1}^{M_{i}} \tilde{G}_{i k s}\left(X_{i} ; t\right) Y_{s}^{(i)}+\tilde{\Phi}_{i k}\left(X_{i} ; t\right), \quad i, k=1,2, \ldots, N
$$

and

$$
e^{t X_{i}} Y_{k}^{(i)} e^{-t X_{i}}=\sum_{s=1}^{N} \tilde{U}_{i k s}\left(X_{i} ; t\right) X_{s}+\sum_{s=1}^{M_{i}} \tilde{V}_{i k s}\left(X_{i} ; t\right) Y_{s}^{(i)}+\tilde{W}_{i k}\left(X_{i} ; t\right)
$$

where the functions $\tilde{F}_{i k s}(x ; t), \ldots, \tilde{W}_{i k}(x ; t)$ are obtained from the polynomials $F_{i k s}^{(n)}(x), \ldots$, $W_{i k}^{(n)}(x)$ by an obvious way, e.g.

$$
\tilde{F}_{i k s}(x ; t)=\sum_{n=0}^{\infty} \frac{t^{n}}{n !} F_{i k s}^{(n)}(x)
$$

etc.

Thus, as in the previous section, we have an explicit linear Heisenberg evolution of the operators $X_{i}, Y_{k}^{(i)}$ under the action of $N$ Hamiltonians $X_{i}, i=1,2, \ldots, N$. In contrast to the previous section, the operators $Y_{k}^{(i)}$ do not in general provide the linear evolution, i.e. they cannot be chosen as Hamiltonians. They serve only as an auxiliary tool in our picture.

The total number $M$ of extension operators $Y_{k}^{(i)}$ can be less then $M_{1}+M_{2}+\cdots+M_{N}$, because in special cases some of these operators can coincide, as we will see later.

It will be convenient to call the corresponding algebras the quasi-linear algebras of the type $(N, M)$, where $N$ is number of the "true Hamiltonians" $X_{1}, \ldots, X_{N}$ and $M$ is a total number of extension operators $Y_{k}^{(i)}$. For quasi-linear algebras without extension we will use the symbol $(N, 0)$.

Of course, essentially the same picture is valid in classical case if one replaces the commutators $[\cdots]$ with the Poisson brackets $\{\cdots\}$.

In the next sections we construct simplest examples of the quasi-linear algebras in both classical and quantum (operator) pictures. 


\section{The $q$-oscillator algebra}

Consider the simplest case when we have only two operators $X, Y$ (i.e. $N=2$ ) and we demand that property (2.1) will be satisfied when $H$ is chosen as either $X$ or $Y$, i.e. in this case we have the quasi-linear algebra of type $(2,0)$ without extension. From previous considerations it follows that two conditions

$$
[X, Y]=F_{1}(Y) X+\Phi_{1}(Y), \quad[Y, X]=F_{2}(X) Y+\Phi_{2}(X)
$$

should be valid, where $F_{i}(z), \Phi_{i}(z), i=1,2$ are some functions.

In the classical case we will have similar conditions:

$$
\{x, y\}=F_{1}(y) x+\Phi_{1}(y), \quad\{y, x\}=F_{2}(x) y+\Phi_{2}(x)
$$

with commutators $[\cdots]$ replaced with the Poisson brackets $\{\cdots\}$. We see that necessary condition for compatibility of (4.1) or (4.2) is

$$
F_{1}(Y) X+\Phi_{1}(Y)+F_{2}(X) Y+\Phi_{2}(X)=0 .
$$

In the classical case (i.e. when $X, Y$ are two independent commuting variables) we have a simple functional equation for 4 unknown functions $F_{i}(z), \Phi_{i}(z), i=1,2$. Assume first that the function $F_{2}(x)$ is not a constant. Then taking two distinct values $x=\alpha$ and $x=\beta$ and considering (4.3) as a system for unknowns $F_{1}(y), \Phi_{1}(y)$ we easily find that these functions should be linear in $y$. Quite similarly, we find that functions $F_{2}(x), \Phi_{2}(x)$ should be linear as well. After simple calculations we obtain in this case the most general solution

$$
\{x, y\}=\alpha x y+\beta_{1} x+\beta_{2} y+\gamma
$$

where $\alpha, \beta_{1}, \beta_{2}, \gamma$ are arbitrary constants $(\alpha \neq 0)$. By an appropriate affine transformation of the variables $x \rightarrow \xi_{1} x+\eta_{1}, y \rightarrow \xi_{2} y+\eta_{2}$ we can reduce (4.4) to a canonical form

$$
\{x, y\}=\alpha x y-1
$$

in the case if the polynomial $\alpha x y+\beta_{1} x+\beta_{2} y+\gamma$ is irreducible. If this polynomial is reducible (i.e. can be presented as a product of two linear polynomials in $x, y$ ) then we have the canonical form

$$
\{x, y\}=\alpha x y
$$

In quantum case we have correspondingly either algebra of the form

$$
[X, Y]=\alpha X Y+\gamma
$$

if $\gamma \neq 0$, or the Weyl operator pair

$$
X Y=q Y X
$$

Note that relation (4.5) can be rewritten in other canonical form

$$
X Y-q Y X=1
$$

with some parameter $q \neq 0,1$. The pair of operators $X, Y$ satisfying the commutation relation (4.6) is called the $q$-oscillator algebra. 
In this case the Heisenberg evolution becomes very simple. Indeed, consider, e.g. $Y$ as the Hamiltonian. We have from (4.6)

$$
[Y,[Y, X]]=\omega^{2} Y^{2} X-\omega Y
$$

where $\omega=1-q$, and, by induction,

$$
a d_{Y}^{n} X=\omega^{n} Y^{n} X-\omega^{n-1} Y^{n-1}, \quad n=1,2, \ldots
$$

Hence we have explicitly

$$
e^{t Y} X e^{-t Y}=e^{\omega t Y} X-\phi(Y ; t),
$$

where

$$
\phi(y ; t)=\int_{0}^{t} e^{\omega \tau y} d \tau=\frac{e^{\omega t y}-1}{\omega y} .
$$

When $\omega \rightarrow 0$ (i.e. $q \rightarrow 1$ ) one obtains

$$
e^{t Y} X e^{-t Y}=X-t+\omega t Y(X-t / 2)+O\left(\omega^{2}\right) .
$$

Here the first term $X-t$ in the right-hand side corresponds to the simple Weyl shift of the harmonic oscillator. Indeed, when $X, Y$ satisfy the Heisenberg-Weyl commutation relation $[X, Y]=1$ then

$$
e^{t Y} X e^{-y Y}=X-t,
$$

i.e. the operator $X$ is shifted by a constant under action of the Hamiltonian $H=Y$. The second term $\omega t Y(X-t / 2)$ describes a small " $q$-deformation" of the Weyl shift.

\section{The Askey-Wilson algebra}

The Askey-Wilson algebra $A W(3)[8,28]$ can be presented in several equivalent forms. One of them consists of 3 operators $K_{1}, K_{2}, K_{3}$ with the commutation relations

$$
\begin{aligned}
& {\left[K_{1}, K_{2}\right]=K_{3},} \\
& {\left[K_{2}, K_{3}\right]=2 \rho K_{2} K_{1} K_{2}+a_{1}\left\{K_{1}, K_{2}\right\}+a_{2} K_{2}^{2}+c_{1} K_{1}+d K_{2}+g_{1},} \\
& {\left[K_{3}, K_{1}\right]=2 \rho K_{1} K_{2} K_{1}+a_{2}\left\{K_{1}, K_{2}\right\}+a_{1} K_{1}^{2}+c_{2} K_{2}+d K_{1}+g_{2}}
\end{aligned}
$$

with some constants $\rho, a_{1}, a_{2}, c_{1}, c_{2}, d, g_{1}, g_{2}$. The symbol $\{\cdots\}$ stands for the anticommutator: $\{X, Y\} \equiv X Y+Y X$.

The $A W(3)$ algebra belongs to quasi-linear type of $(2,1)$. Indeed, we can rewrite it in the form

$$
\begin{aligned}
& {\left[K_{1}, K_{2}\right]=K_{3},} \\
& {\left[K_{2},\left[K_{2}, K_{1}\right]\right]=R_{2}\left(K_{2}\right) K_{1}+R_{1}\left(K_{2}\right) K_{3}+R_{0}\left(K_{2}\right),} \\
& {\left[K_{1},\left[K_{1}, K_{2}\right]\right]=S_{2}\left(K_{1}\right) K_{2}+S_{1}\left(K_{1}\right) K_{3}+S_{0}\left(K_{1}\right),}
\end{aligned}
$$

where

$$
\begin{array}{ll}
R_{2}(x)=-2 \rho x^{2}-2 a_{1} x-c_{1}, & R_{1}(x)=-2 \rho x-a_{1}, \quad R_{0}(x)=-a_{2} x^{2}-d x-g_{1}, \\
S_{2}(x)=-2 \rho x^{2}-2 a_{2} x-c_{2}, & S_{1}(x)=2 \rho x+a_{2}, \quad S_{0}(x)=-a_{1} x^{2}-d x-g_{2} .
\end{array}
$$


We see that $A W(3)$ algebra in the form (5.2) has quasi-linear structure with $N=2, M=1$. The operators $K_{1}, K_{2}$ play the role of the true Hamiltonians whereas the operator $K_{3}$ is the only extension.

If $\rho \neq 0$ one can present the same algebra in an equivalent (more symmetric) form in terms of operators $X, Y, Z[28,24,27]$

$$
X Y-q Y X=Z+C_{3}, \quad Y Z-q Z Y=X+C_{1}, \quad Z X-q X Z=Y+C_{2},
$$

or, in a pure commutator form

$$
\begin{aligned}
& {[X, Y]=(q-1) Y X+Z+C_{3}, \quad[Y, Z]=(q-1) Z Y+X+C_{1},} \\
& {[Z, X]=(q-1) X Z+Y+C_{2} .}
\end{aligned}
$$

In this form we have quasi-linear algebra of type $(3,0)$ without extension, i.e. in this case all 3 operators $X, Y, Z$ of the algebra play the role of the Hamiltonians. Hence in this case the linearity property holds for all operators $X, Y, Z$.

It is instructive to derive directly transformation property of, say, operator $X$ under Hamiltonian action of the operator $Y$. We have

$$
\operatorname{ad}_{Y}^{n} X=U_{n}(Y) X+V_{n}(Y) Z+W_{n}(Y)
$$

where $U_{n}(x), V_{n}(x), W_{n}(x)$ are polynomials in $x$. Initial conditions are obvious:

$$
U_{0}(x)=1, \quad V_{0}(x)=W_{0}(x)=0 .
$$

Derive recurrence relations for the polynomials $U_{n}(x), V_{n}(x), W_{n}(x)$. For this goal let as apply the operator $\operatorname{ad}_{Y}$ with respect to (5.4):

$$
\begin{aligned}
\operatorname{ad}_{Y}^{n+1} X= & U_{n}(Y)[Y, X]+V_{n}(Y)[Y, Z]=\left((1-q) Y U_{n}(Y)+q^{-1} V_{n}(Y)\right) X \\
& +\left(\left(1-q^{-1}\right) Y V_{n}(Y)-U_{n}(Y)\right) Z-C_{3} U_{n}(Y)+q^{-1} C_{1} V_{n}(Y) .
\end{aligned}
$$

Hence we have

$$
\begin{aligned}
& U_{n+1}=(1-q) Y U_{n}+q^{-1} V_{n}, \quad V_{n+1}=-U_{n}+\left(1-q^{-1}\right) Y V_{n}, \\
& W_{n+1}=-C_{3} U_{n}+C_{1} q^{-1} V_{n} .
\end{aligned}
$$

From recurrence relations (5.5) it is possible to find polynomials $U_{n}, V_{n}, W_{n}$ explicitly. In particular, it is clear that $U_{n}(Y)$ is a polynomial of degree $n$ and $V_{n}(Y), W_{n}(Y)$ are polynomials of degree $n-1$.

For the transformed operator $X(t)$ we have

$$
X(t)=e^{t Y} X e^{-t Y}=E_{1}(t ; Y) X+E_{2}(t ; Y) Z+E_{0}(t ; Y),
$$

where

$$
E_{1}(t ; Y)=\sum_{n=0}^{\infty} \frac{t^{n} U_{n}(Y)}{n !}, \quad E_{2}(t ; Y)=\sum_{n=0}^{\infty} \frac{t^{n} V_{n}(Y)}{n !}, \quad E_{0}(t ; Y)=\sum_{n=0}^{\infty} \frac{t^{n} W_{n}(Y)}{n !} .
$$

From (5.5) we have a system of linear differential equations for unknown functions $E_{1}(t), E_{2}(t)$

$$
\dot{E}_{1}(t ; x)=(1-q) x E_{1}+q^{-1} E_{2}, \quad \dot{E}_{2}(t ; x)=-E_{1}+\left(1-q^{-1}\right) x E_{2} .
$$

Initial conditions are $E_{1}(0)=1, E_{2}(0)=0$. This system has constant coefficients (not depending on $t$ ) and hence can be elementary integrated:

$$
E_{1}(t ; x)=a_{11}(x) e^{\omega_{1}(x) t}+a_{12}(x) e^{\omega_{2}(x) t}, \quad E_{2}(t ; x)=a_{21}(x) e^{\omega_{1}(x) t}+a_{22}(x) e^{\omega_{2}(x) t},
$$


where $\omega_{1,2}(x)$ are roots of the characteristic equation

$$
\omega^{2}+x\left(2-q-q^{-1}\right)(x-\omega)+q^{-1}=0
$$

and the coefficients $a_{i k}(x)$ can be found by standard methods.

The function $E_{0}(t ; x)$ can then be found as

$$
E_{0}(t ; x)=-C_{3} \int_{0}^{t} E_{1}(\tau ; x) d \tau+C_{1} q^{-1} \int_{0}^{t} E_{2}(\tau ; x) d \tau
$$

We see that the Heisenberg evolution is described by elementary functions (linear combinations of exponents) in the argument $t$. But the coefficients in these linear combinations depend on operator $H$.

Similar expressions are valid for all other possible choices of the Hamiltonian (i.e. $H=X$ or $H=Z$ ) due to symmetric form of algebra (5.3).

Thus for $\rho \neq 0$ essentially the same $A W(3)$ algebra can be presented in two equivalent forms either as an algebra of type $(2,1)$ with the only extension or as an algebra of type $(3,0)$ without extension. However if $\rho=0$ then the only type $(2,1)$ is known (this special case corresponds to the so-called quadratic Racah algebra QR(3) [8]).

So far, we have no general results in quantum (i.e. operator) case concerning classification scheme. In particular, we do not know, whether $A W(3)$ algebra is the only quasi-linear algebra of type $(2,1)$ or $(3,0)$. In the classical case the situation is slightly better: we already showed that for the type $(2,0)$ the only $q$-oscillator algebra appears. In the next section we consider a classification scheme for the type $(3,0)$.

\section{Classical case of type $(3,0)$}

Consider the classical case of quasi-linear algebras of type $(3,0)$. This means that we have 3 dynamical variables $x, y, z$ satisfying the Poisson bracket relations

$$
\{y, z\}=F_{1}(x, y, z), \quad\{z, x\}=F_{2}(x, y, z), \quad\{x, y\}=F_{3}(x, y, z),
$$

where the functions $F_{i}(x, y, z)$ should be chosen in such way to satisfy the Jacobi identity

$$
\{\{x, y\}, z\}+\{\{y, z\}, x\}+\{\{z, x\}, y\}=0 .
$$

It is easily seen that this condition is equivalent to the relation [6]

$$
(\mathbf{F}, \operatorname{rot} \mathbf{F})=0,
$$

where the vector $\mathbf{F}$ has Cartesian components $\left(F_{1}, F_{2}, F_{3}\right)$ and rot is standard differentiation curl operator acting on the vector $\mathbf{F}$.

Relation (6.1) has an obvious solution

$$
\mathbf{F}=\nabla Q(x, y, z),
$$

where $\nabla$ is the gradient operator and $Q(x, y, z)$ is a function of 3 variables. In this case we have the defining Poisson relations in the form

$$
\{y, z\}=Q_{x}, \quad\{z, x\}=Q_{y}, \quad\{x, y\}=Q_{z},
$$

where $Q_{x}$ means derivation with respect to $x$ etc. Note that in this case the function $Q(x, y, z)$ is the Casimir element of the algebra, i.e. $\{x, Q\}=\{y, Q\}=\{z, Q\}=0$. Poisson brackets of such 
types are sometimes called the Nambu brackets or Mukai-Sklyanin algebras [17]. However, the Nambu bracket (6.2) do not exhaust all admissible Poisson algebras with 3 generators. There are non-trivial examples corresponding to $\operatorname{rot} \mathbf{F} \neq 0$.

Consider restrictions on the functions $F_{i}(x, y, z)$ coming from the quasi-linear property.

On the one side, choosing $y$ to be a Hamiltonian, we have by this property

$$
\{x, y\}=F_{3}(x, y, z)=\Phi_{1}(y) x+\Phi_{2}(y) z+\Phi_{3}(y) .
$$

On the other side, choosing $x$ to be a Hamiltonian, we have analogously

$$
\{x, y\}=\Phi_{4}(x) y+\Phi_{5}(x) z+\Phi_{6}(x)
$$

with some functions $\Phi_{i}(x), i=1,2, \ldots, 6$.

From (6.4) and (6.5) we see that $F_{3}(x, y, z)$ should be a polynomial having degree no more than 1 with respect to each variable $x, y, z$ and the most general form of this polynomial is

$$
F_{3}(x, y, z)=\alpha_{3} x y+\beta_{31} x+\beta_{32} y+\beta_{33} z+\gamma_{3} .
$$

Quite analogously, taking functions $F_{1}, F_{2}$ we obtain

$$
F_{i}(x, y, z)=\alpha_{i} x_{k} x_{l}+\sum_{s=1}^{3} \beta_{i s} x_{s}+\gamma_{i},
$$

where $x_{1}=x, x_{2}=y, x_{3}=z$ and $\alpha_{i}, \beta_{i s}, \gamma_{i}$ are some constants. As usual in rhs of (6.6) notation $x_{k} x_{l}$ means that triple $(i, k, l)$ has no coinciding entries.

Condition (6.1) imposes strong restrictions upon the coefficients $\alpha_{i}, \beta_{i k}, \gamma_{i}$. It is convenient to analyze the canonical forms of obtained algebras up to affine transformations $x_{i} \rightarrow \xi_{i} x_{i}+\eta_{i}$ with some constants $\xi_{i}, \eta_{i}$ (of course we demand that $\xi_{1} \xi_{2} \xi_{3} \neq 0$ ).

(i) If diagonal entries $\beta_{i i}$ are all nonzero then necessarily $\alpha_{1}=\alpha_{2}=\alpha_{3}=\alpha$. We will assume $\alpha \neq 0$ (otherwise we will obtain the Lie-Poisson algebras). Then the matrix $\beta$ should be symmetric: $\beta_{i k}=\beta_{k i}$. These conditions are also sufficient for validity of the Jacobi identity. After an appropriate affine transformation we reduce our algebra to the canonical form

$$
F_{i}(x, y, z)=\alpha x_{k} x_{l}+x_{i}+\gamma_{i}
$$

with only 4 independent free parameters: $\alpha$ and $\gamma_{i}, i=1,2,3$. This is exactly the classical version of the Askey-Wilson algebra $A W(3)$ [12].

(ii) If one of diagonal entries is zero, say $\beta_{33}=0$, then again we have the same necessary and sufficient conditions $\alpha_{1}=\alpha_{2}=\alpha_{3}=\alpha \neq 0$ and $\beta_{i k}=\beta_{k i}$. The canonical form is

$$
F_{1}=\alpha x_{2} x_{3}+x_{1}+\gamma_{1}, \quad F_{2}=\alpha x_{1} x_{3}+x_{2}+\gamma_{2}, \quad F_{3}=\alpha x_{2} x_{1}+\gamma_{3} .
$$

In both cases (i) and (ii) we have the Nambu-Poisson brackets (6.3) with

$$
Q=\alpha x y z+\left(x^{2}+y^{2}+z^{2}\right) / 2+\gamma_{1} x+\gamma_{2} y+\gamma_{3} z
$$

for the case $(i)$ and

$$
Q=\alpha x y z+\left(x^{2}+y^{2}\right) / 2+\gamma_{1} x+\gamma_{2} y+\gamma_{3} z
$$

for the case $(i i)$.

(iii) Two diagonal entries are zero, say $\beta_{22}=\beta_{33}=0$ and $\beta_{11} \neq 0$. Then there exist 2 possibilities: in the first case $\alpha_{1}=\alpha_{2}=\alpha_{3}=\alpha \neq 0$ and the canonical form of the algebra is

$$
F_{1}=\alpha x_{2} x_{3}+x_{1}+\gamma_{1}, \quad F_{2}=\alpha x_{1} x_{3}+\gamma_{2}, \quad F_{3}=\alpha x_{2} x_{1}+\gamma_{3} .
$$


This is again the algebra of Nambu type with

$$
Q=\alpha x y z+x^{2} / 2+\gamma_{1} x+\gamma_{2} y+\gamma_{3} z .
$$

(iv) As in the previous case we have $\beta_{22}=\beta_{33}=0$ and $\beta_{11} \neq 0$. But now there is a possibility of noncoinciding entries $\alpha_{i}$. Namely we have $\alpha_{2}=\alpha_{3}$ but $\alpha_{1} \neq \alpha_{2}$. We will assume that $\alpha_{2} \alpha_{1} \neq 0$. Then we have the canonical form

$$
F_{1}=\alpha_{1} x_{2} x_{3}+x_{1}, \quad F_{2}=\alpha_{2} x_{1} x_{3}, \quad F_{3}=\alpha_{2} x_{2} x_{1} .
$$

This case does not belong to the Nambu type.

(v) There is further degeneration of the previous case. Namely we can allow $\alpha_{1}=0, \alpha_{2} \neq 0$ or $\alpha_{2}=0, \alpha_{1} \neq 0$. In the first case we have the canonical form

$$
F_{1}=x_{1}+\gamma_{1}, \quad F_{2}=\alpha_{2} x_{1} x_{3}, \quad F_{3}=\alpha_{2} x_{2} x_{1} .
$$

In the second case the canonical form is

$$
F_{1}=\alpha_{1} x_{2} x_{3}+x_{1}+\gamma_{1}, \quad F_{2}=x_{3}, \quad F_{3}=x_{2} .
$$

(vi) Assume that all diagonal entries are zero $\beta_{11}=\beta_{22}=\beta_{33}=0$. Then if $\alpha_{1} \alpha_{2} \alpha_{3} \neq 0$ we have the canonical form

$$
F_{1}=\alpha_{1} x_{2} x_{3}, \quad F_{2}=\alpha_{2} x_{1} x_{3}, \quad F_{3}=\alpha_{3} x_{2} x_{1} .
$$

It is interesting to note that obtained algebras of types (i)-(vi) correspond to the list of so-called 3-dimensional skew-polynomial algebras introduced by Bell and Smith [22, 19]. In our case we have classical (i.e. Poisson brackets) analogues of the 3-dimensional skew-polynomial algebras.

Concerning other aspects and applications of the classical (Poissonic) version of the $A W(3)$ algebra see e.g. [12].

\section{Beyond the AW-algebra}

Consider the so-called Dolan-Grady relations [5] for two operators $A_{0}, A_{1}$

$$
\left[A_{0},\left[A_{0},\left[A_{0}, A_{1}\right]\right]\right]=\omega^{2}\left[A_{0}, A_{1}\right], \quad\left[A_{1},\left[A_{1},\left[A_{1}, A_{0}\right]\right]\right]=\omega^{2}\left[A_{1}, A_{0}\right],
$$

where $\omega$ is an arbitrary constant. These relations generate the so-called infinite-dimensional Onsager algebra [4] which plays a crucial role in algebraic solution of the Ising model as well as of some more general models in statistical physics [18].

On the other hand, we can consider the DG-relations from the "quasi-linear" algebras point of view. Indeed, introduce the operators

$$
A_{2}=\left[A_{0}, A_{1}\right], \quad A_{3}=\left[A_{0},\left[A_{0}, A_{1}\right]\right], \quad A_{4}=\left[A_{1},\left[A_{1}, A_{0}\right]\right] .
$$

Then we see that

$$
\begin{array}{lrr}
\operatorname{ad}_{A_{0}}^{2 n+2} A_{1}=\omega^{2 n} A_{3}, & \operatorname{ad}_{A_{0}}^{2 n+1} A_{1}=\omega^{2 n} A_{2}, & n=0,1,2, \ldots, \\
\operatorname{ad}_{A_{1}}^{2 n+2} A_{0}=\omega^{2 n} A_{4}, & \operatorname{ad}_{A_{1}}^{2 n+1} A_{0}=-\omega^{2 n} A_{2}, & n=0,1,2, \ldots, \\
\operatorname{ad}_{A_{0}}^{2 n+2} A_{2}=\omega^{2 n+2} A_{2}, & \operatorname{ad}_{A_{0}}^{2 n+1} A_{2}=\omega^{2 n} A_{3}, & n=0,1,2, \ldots, \\
\operatorname{ad}_{A_{1}}^{2 n+2} A_{2}=\omega^{2 n+2} A_{2}, & \operatorname{ad}_{A_{1}}^{2 n+1} A_{2}=-\omega^{2 n} A_{4}, & n=0,1,2, \ldots
\end{array}
$$


From these formulas we obtain an explicit Heisenberg evolution of the operators $A_{0}, A_{1}, A_{2}$ if the operators $A_{0}$ or $A_{1}$ are chosen as Hamiltonians:

$$
\begin{aligned}
& e^{t A_{0}} A_{1} e^{-t A_{0}}=A_{1}+\frac{\sinh (\omega t)}{\omega} A_{2}+\frac{\operatorname{coth}(\omega t)-1}{\omega^{2}} A_{3}, \\
& e^{t A_{0}} A_{2} e^{-t A_{0}}=\cosh (\omega t) A_{2}+\frac{\sinh (\omega t)}{\omega} A_{3}, \\
& e^{t A_{1}} A_{0} e^{-t A_{1}}=A_{0}-\frac{\sinh (\omega t)}{\omega} A_{2}+\frac{\operatorname{coth}(t \omega)-1}{\omega^{2}} A_{4}, \\
& e^{t A_{1}} A_{2} e^{-t A_{1}}=\cosh (\omega t) A_{2}-\frac{\sinh (\omega t)}{\omega} A_{4} .
\end{aligned}
$$

We see that the operators $A_{0}, A_{1}$ can be chosen as Hamiltonians whereas the operators $A_{2}, A_{3}$, $A_{4}$ are the extension. Hence the DG-relations provide an example of a quasi-linear algebra of the type $(2,3)$.

Define now the operator

$$
W=\alpha A_{0}+\beta A_{1}+\gamma A_{2}
$$

with arbitrary parameters $\alpha, \beta, \gamma$. It is seen that

$$
\begin{aligned}
e^{t A_{1}} W e^{-t A_{1}}= & \alpha A_{0}+\beta A_{1}+\left(\gamma \cosh (\omega t)-\alpha \frac{\sinh (\omega t)}{\omega}\right) A_{2} \\
& +\left(\alpha \frac{\cosh (\omega t)-1}{\omega^{2}}-\gamma \frac{\sinh (\omega t)}{\omega}\right) A_{4} .
\end{aligned}
$$

Analogously

$$
\begin{aligned}
e^{\tau A_{0}} W e^{-\tau A_{0}}= & \alpha A_{0}+\beta A_{1}+\left(\gamma \cosh (\omega \tau)+\beta \frac{\sinh (\omega \tau)}{\omega}\right) A_{2} \\
& +\left(\beta \frac{\cosh (\omega \tau)-1}{\omega^{2}}+\gamma \frac{\sinh (\omega \tau)}{\omega}\right) A_{3},
\end{aligned}
$$

where $t$ and $\tau$ are arbitrary parameters. If one chooses

$$
\alpha=\omega \gamma \operatorname{coth}(\omega t / 2), \quad \beta=-\omega \gamma \operatorname{coth}(\omega \tau / 2),
$$

then the terms containing $A_{3}, A_{4}$ disappear and we have

$$
e^{t A_{1}} W e^{-t A_{1}}=\alpha A_{0}+\beta A_{1}-\gamma A_{2}=e^{\tau A_{0}} W e^{-\tau A_{0}},
$$

whence

$$
T W T^{-1}=W,
$$

where

$$
T=e^{-\tau A_{0}} e^{t A_{1}} .
$$

Equivalently, this means that the operator $W$ commutes with the operator $T$ :

$$
T W=W T .
$$

As was noted by Davies [4] this commutation relation was crucial in Onsager's solution of the Ising model, where the operators $T$ play the role of the transfer matrix. We see that this relation follows directly from the quasi-linear property of the DG-relations. 
There is an obvious generalization of the DG-relations preserving the quasi-linear property.

Indeed, define again basic operators $A_{0}, A_{1}$ and their extensions $A_{2}, A_{3}, A_{4}$ by (7.2). But now the triple commutators ad ${ }_{A_{0}}^{3} A_{1}$ and $\operatorname{ad}_{A_{1}}^{3} A_{0}$ can contain not only operator $A_{2}$ as in DG-case (7.1) but arbitrary linear combinations of the type

$$
\left[A_{0},\left[A_{0},\left[A_{0}, A_{1}\right]\right]\right]=g_{1}\left(A_{0}\right) A_{1}+g_{2}\left(A_{0}\right) A_{2}+g_{3}\left(A_{0}\right) A_{3}+g_{0}\left(A_{0}\right)
$$

and

$$
\left[A_{1},\left[A_{1},\left[A_{1}, A_{0}\right]\right]\right]=f_{1}\left(A_{1}\right) A_{0}+f_{2}\left(A_{1}\right) A_{2}+f_{3}\left(A_{1}\right) A_{4}+f_{0}\left(A_{1}\right)
$$

with some polynomials $g_{i}(x), f_{i}(x), i=0, \ldots, 3$. We will call these relations the generalized DGrelations. The ordinary DG-relations corresponds to the choice $g_{0}=f_{0}=g_{1}=f_{1}=g_{3}=f_{3}=0$ and $g_{2}=-f_{2}=\omega^{2}$.

From (7.3) and (7.4) it is obvious that for any positive integer $n$ the repeated commutator $\operatorname{ad}_{A_{0}}^{n} A_{1}$ has the same structure

$$
\operatorname{ad}_{A_{0}}^{n} A_{1}=g_{1}^{(n)}\left(A_{0}\right) A_{1}+g_{2}^{(n)}\left(A_{0}\right) A_{2}+g_{3}^{(n)}\left(A_{0}\right) A_{3}+g_{0}^{(n)}\left(A_{0}\right)
$$

and similarly

$$
\operatorname{ad}_{A_{1}}^{n} A_{0}=f_{1}^{(n)}\left(A_{1}\right) A_{0}+f_{2}^{(n)}\left(A_{1}\right) A_{2}+f_{3}^{(n)}\left(A_{1}\right) A_{4}+f_{0}^{(n)}\left(A_{1}\right)
$$

with polynomials $g_{i}^{(n)}(x)$ and $f_{i}^{(n)}(x)$ can be easily obtained from $g_{i}(x)$ and $f_{i}(x)$. We thus have the "Heisenberg solvability" property

$$
\exp \left(A_{0} t\right) A_{1} \exp \left(-A_{0} t\right)=G_{1}\left(A_{0} ; t\right) A_{1}+G_{2}\left(A_{0} ; t\right) A_{2}+G_{3}\left(A_{0} ; t\right) A_{3}+G_{0}\left(A_{0} ; t\right)
$$

where functions $G_{i}(x ; t)$ can be easily calculated in the same manner as in $(2.3)$. (Similar relation holds for the Heisenberg evolution of the operator $A_{1}$.)

Consider a special example of these generalized DG-relations. The so-called "tridiagonal algebra" proposed by Terwilliger is generated by the two relations [9]

$$
\left[A_{0}, A_{0}^{2} A_{1}+A_{1} A_{0}^{2}-\beta A_{0} A_{1} A_{0}-\gamma\left(A_{0} A_{1}+A_{1} A_{0}\right)-\alpha A_{1}\right]=0
$$

and

$$
\left[A_{1}, A_{1}^{2} A_{0}+A_{0} A_{1}^{2}-\beta A_{1} A_{0} A_{1}-\gamma_{1}\left(A_{0} A_{1}+A_{1} A_{0}\right)-\alpha_{1} A_{0}\right]=0
$$

where $\beta, \gamma, \gamma_{1}, \alpha, \alpha_{1}$ are some constants.

The tridiagonal algebra is closely related with the Askey-Wilson algebra $A W(3)$. Indeed, let the operators $A_{0}, A_{1}$ belong to the AW(3) algebra of the type (5.1). Identify $A_{0}=K_{1}, A_{1}=K_{2}$, $A_{2}=K_{3}$ and rewrite relations (5.1) in an equivalent form getting rid of the operator $K_{3}=A_{2}$ :

$$
\begin{gathered}
A_{1}^{2} A_{0}+A_{0} A_{1}^{2}+2(\rho-1) A_{1} A_{0} A_{1}+a_{1}\left(A_{1} A_{0}+A_{0} A_{1}\right) \\
+a_{2} A_{1}^{2}+c_{1} A_{0}+d A_{1}+g_{1}=0
\end{gathered}
$$

and

$$
\begin{gathered}
A_{0}^{2} A_{1}+A_{1} A_{0}^{2}+2(\rho-1) A_{0} A_{1} A_{0}+a_{2}\left(A_{1} A_{0}+A_{0} A_{1}\right) \\
\quad+a_{1} A_{0}^{2}+c_{2} A_{1}+d A_{0}+g_{2}=0
\end{gathered}
$$


Now applying the operator $\operatorname{ad}_{A_{0}}$ to (7.8) we obtain relation (7.5). Analogously, applying the operator $\operatorname{ad}_{A_{1}}$ to (7.7) we obtain relation (7.6). Parameters of the tridiagonal algebra (7.5), (7.6) are related with parameters of the $A W(3)$ algebra as follows

$$
\beta=2(1-\rho), \quad \alpha=-c_{2}, \quad \alpha_{1}=-c_{1}, \quad \gamma=-a_{2}, \quad \gamma_{1}=-a_{1} .
$$

Thus the tridiagonal algebra (7.5), (7.6) follows from the $A W(3)$ algebra (5.1). However the reciprocal statement is not valid: the tridiagonal algebra is larger than $A W(3)$ [9].

It is easily verified that the tridiagonal algebra (7.5), (7.6) can be presented in an equivalent form:

$$
\begin{aligned}
& {\left[A_{0},\left[A_{0},\left[A_{0}, A_{1}\right]\right]\right]=(2-\beta)\left(A_{0} A_{3}-A_{0}^{2} A_{2}\right)+2 \gamma A_{0} A_{2}-\gamma A_{3}+\alpha A_{2},} \\
& {\left[A_{1},\left[A_{1},\left[A_{1}, A_{0}\right]\right]\right]=(2-\beta)\left(A_{1} A_{4}+A_{1}^{2} A_{2}\right)-2 \gamma_{1} A_{1} A_{2}-\gamma_{1} A_{4}-\alpha_{1} A_{2},}
\end{aligned}
$$

where the operators $A_{2}, A_{3}, A_{4}$ are defined as (7.2). Comparing (7.9) with (7.3) and (7.4) we see that the tridiagonal algebra (7.5) indeed belongs to the quasi-linear type with the coefficients

$$
\begin{aligned}
& g_{0}=g_{1}=0, \quad g_{2}(x)=(\beta-2) x^{2}+2 \gamma x+\alpha, \quad g_{3}(x)=(2-\beta) x-\gamma, \\
& f_{0}=f_{1}=0, \quad f_{2}(x)=(2-\beta) x^{2}-2 \gamma_{1} x-\alpha_{1}, \quad f_{3}(x)=(2-\beta) x-\gamma_{1} \text {. }
\end{aligned}
$$

As in the case of the ordinary DG-relations, the tridiagonal algebra is the quasi-linear algebra with two basic operators $A_{0}, A_{1}$ and 3 extensions $A_{2}, A_{3}, A_{4}$, i.e. it has the same type $(2,3)$ as the DG-algebra.

In a special case $\gamma=\gamma_{1}=0$ we obtain the so-called $q$-deformation of the Dolan-Grady relations $[24,25]$ :

$$
\left[A_{0},\left[A_{0},\left[A_{0}, A_{1}\right]_{q}\right]_{q^{-1}}\right]=\alpha\left[A_{0}, A_{1}\right], \quad\left[A_{1},\left[A_{1},\left[A_{1}, A_{0}\right]_{q}\right]_{q^{-1}}\right]=\alpha_{1}\left[A_{1}, A_{0}\right]
$$

where $[X, Y]_{q}=q^{1 / 2} X Y-q^{-1 / 2} Y X$ is so-called $q$-commutator. The parameter $q$ is connected with the parameter $\beta$ by the relation $\beta=q+q^{-1}$. This deformed DG-algebra plays an important role in theory of quantum $X X Z$ Heisenberg model, Azbel-Hofstadter model etc [3, 2].

It would be interesting to study what is the meaning of the exact solvability of the Heisenberg picture for the $q$-deformed DG-relations (7.10) in corresponding exactly solvable models.

\section{Quasi-linear algebras and exactly solvable systems}

Recently Odake and Sasaki proposed an interesting approach to exactly solvable classical and quantum mechanical models in the Heisenberg picture $[15,16]$. They noticed that almost all exactly solvable one-dimensional quantum models admit exact solution in both Schrödinger and Heisenberg pictures.

Consider, e.g. the one-dimensional model described by the standard one-dimensional Hamiltonian

$$
H=p^{2} / 2+U(x)
$$

where $p$ is the momentum operator and $U(x)$ is a potential. Of course, the standard Heisenberg commutation relation between coordinate and momentum operators $[x, p]=i$ is assumed.

Introduce the operator $X=f(x)$ with some function $f(x)$ and consider the Heisenberg evolution of this operator under the Hamiltonian $H$ :

$$
X(t)=\exp (i H t) X \exp (-i H t)=X+i t[H, X]-\frac{t^{2}}{2}[H,[H, X]]+\cdots+\frac{(i t)^{n}}{n !} \operatorname{ad}_{H}^{n} X+\cdots
$$


In general for arbitrary Hamiltonian $H$ the structure of the expression $\operatorname{ad}_{H}^{n} X$ will be too complicated, and so it is hopeless to find explicit solution in the Heisenberg picture. Assume, however, that the Hamiltonian $H$ and the function $f(x)$ satisfy the restriction

$$
[H,[H, X]]=\operatorname{ad}_{H}^{2} X=g_{1}(H) X+g_{2}(H) Y+g_{0}(H),
$$

with some functions $g_{i}(H), i=0,1,2$, where we introduce the operator

$$
Y=[H, X]=-2 i f^{\prime}(x)-f^{\prime \prime}(x) .
$$

Then it is obvious that ansatz (8.2) (proposed by Odake and Sasaki $[15,16]$ ) leads to an explicit solution

$$
X(t)=G_{1}(H ; t) X+G_{2}(H ; t) Y+G_{0}(H ; t),
$$

where the functions $G_{i}(H ; t), i=0,1,2$ have elementary behavior in time $t$ (i.e. they can be expressed in terms of trigonometric or hyperbolic functions).

Simple considerations (see [15]) lead to the conclusion that $g_{1}(H)$ and $g_{0}(H)$ should be linear polynomials in $H$ whereas $g_{2}(H)$ should be a constant such that $2 g_{2}=-g_{1}^{\prime}(H)=-\alpha_{1}$ with some constant $\alpha_{1}$.

It is easy to find (see [15]) that the most general function $f(x)$ satisfying this property is a solution of the equation

$$
f^{\prime \prime}(x)=-\frac{1}{2}\left(\alpha_{1} f(x)+\beta_{1}\right)
$$

with arbitrary constants $\alpha_{1}, \beta_{1}$. Hence $f(x)$ is an elementary function: it is either a quadratic polynomial in $x$, or a superposition of two exponents (plus a constant). Corresponding potentials $U(x)$ can also be easily found [15] - they coincide with well-known "exactly-solvable" potentials in quantum mechanics:

(i) singular oscillator:

$$
U(x)=a_{1} x^{2}+a_{2} x^{-2}+a_{0} ;
$$

(ii) the Morse potential

$$
U(x)=a_{1} e^{-2 x}+a_{2} e^{-x}+a_{0} ;
$$

(iii) the Pöschl-Teller potential

$$
U(x)=a_{1} \sin ^{-2} x+a_{2} \cos ^{-2} x+a_{0}
$$

(in the latter case one can replace trigonometric functions with hyperbolic ones that gives hyperbolic analogs of the Pöschl-Teller potential).

Observe now that two operators $H, X$ together with the third operator $Y=[H, X]$ form some quasi-linear algebra. In this case the operator $Y$ plays the role of extension. Indeed, we already established the first commutation relation (8.2) which in our case can be rewritten in the form

$$
[H, Y]=\left(\alpha_{1} H+\alpha_{0}\right) X-\frac{\alpha_{1}}{2} Y+\beta_{1} H+\beta_{0} .
$$

The second commutation relation is verified easily:

$$
[Y, X]=-f^{\prime 2}(x)=\frac{\alpha_{1}}{2} X^{2}+\beta_{1} X+\varepsilon
$$


with some constant $\varepsilon$. Relations (8.3) and (8.4) show that we have a quasi-linear algebra of type $(2,1)$ with two Hamiltonians $H, X$ and the only extension $Y$. This algebra is equivalent to so-called quadratic Jacobi algebra $Q J(3)$ which was considered in [8] as a hidden symmetry algebra of exactly solvable Schrödinger Hamiltonians (see also [13]). The Jacobi algebra is a special case of the algebra $A W(3)$ [8]. It corresponds to the following choice of the parameters $\rho=a_{2}=d=0$ in (5.1). We thus see that the Odake-Sasaki approach $[15,16]$ for the Schrödinger Hamiltonians $H$ (8.1) leads naturally to the Jacobi algebra $Q J(3)$.

Consider a difference analogue of the Schrödinger Hamiltonian. We choose

$$
H=A(s) T^{+}+C(s) T^{-}+B(s),
$$

where operator $H$ acts on the space of functions $f(s)$ depending on a variable $s$ and operators $T^{ \pm}$ are the standard shift operators

$$
T^{ \pm} f(s)=f(s \pm 1) .
$$

The operator $H$ is a second-order difference operator. Hence the eigenvalue equation

$$
H f(s)=\lambda f(s)
$$

can be considered as a difference analogue of the corresponding one-dimensional Schrödinger equation.

Introduce also the operators $X$ which acts as a multiplication

$$
X f(s)=x(s) f(s)
$$

with some function $x(s)$ which will be called a "grid" and the commutator $Y=[H, X]$.

We now would like to find when the relation

$$
\operatorname{ad}_{H}^{2} X=[H, Y]=W_{1}(H) X+W_{2}(H) Y+W_{0}(H)
$$

holds, where $W_{i}(H), i=0,1,2$ are some polynomials in $H$. If (8.6) is fulfilled then obviously we have an explicit time dynamics of the "grid" operator $X$ under the Heisenberg evolution with the Hamiltonian $H$ :

$$
X(t) \equiv \exp (H t) X \exp (-H t)=Q_{1}(H ; t) X+Q_{2}(H ; t) Y+Q_{0}(H ; t)
$$

with functions $Q_{i}(H ; t)$ which can be easily explicitly calculated from $W_{i}(H)$.

We will assume that $A(s) C(s) \neq 0$ and the grid $x(s)$ is nondegenerated, i.e. $x(s) \neq x(s+1)$ and $x(s) \neq x(s+2)$. Under such conditions it is always possible to assume that $C(s)=1$. Indeed, using similarity transformation $H \rightarrow F(s) H F^{-1}(s)$ with some functions $F(s)$ one can achieve the condition $C(s)=1$. Such similarity transformation does not change the operator relation (8.6).

Assume that polynomial $W_{2}(H)$ have a degree $N$ with respect to the variable $H$. Then it is clear from nondegeneracy of $x(s)$ that degrees of polynomials $W_{1}(H)$ and $W_{0}(H)$ cannot exceed $N+1$. Assuming that $\operatorname{deg}\left(W_{1}\right)=\operatorname{deg}\left(W_{0}\right)=N+1$ (otherwise we again obtain degeneracy of $x(s)$ ) we obtain from (8.6) an operator identity of the form

$$
\sum_{k=-N-1}^{N+1} E_{k}(s) T^{k}=0
$$

with coefficients $E_{k}(s)$ which can be explicitly calculated after substitution of (8.5) and $X=x(s)$ into (8.6). From this identity we should have

$$
E_{k}(s) \equiv 0
$$


for all $k=-N-1,-N, \ldots, N+1$. Assume that $N \geq 2$. Then the highest-order conditions $E_{-N-1}(s)=E_{N+1}(s) \equiv 0$ are reduced to very simple first-degree recurrence relations for the grid $x(s)$ :

$$
\xi_{N} x(s+1)+\eta_{N} x(s)+\zeta_{N}=0
$$

and

$$
\xi_{N} x(s-1)+\eta_{N} x(s)+\zeta_{N}=0,
$$

where $\xi_{N}, \eta_{N}, \zeta_{N}$ are some complex coefficients not depending on $s$. It is easily verified that for all possible choices of the coefficients these conditions are incompatible with nondegeneracy conditions $x(s) \neq x(s+1)$ and $x(s-1) \neq x(s+1)$ for the grid $x(s)$. Hence necessarily $N \leq 1$. Thus $W_{2}(H)$ should be a linear polynomial (or a constant) in $H$ and $W_{0}(H), W_{1}(H)$ should be quadratic (or linear) polynomials in $H$. Consider again the highest-order conditions $E_{2}(s)=E_{-2}(s) \equiv 0$ :

$$
\xi x(s+1)+\eta x(s)+x(s-1)+\zeta=0
$$

and

$$
\xi x(s-1)+\eta x(s)+x(s+1)+\zeta=0
$$

with some constants $\xi, \eta, \zeta$.

These conditions are compatible with non-degeneracy conditions for the grid $x(s)$ only if $\xi=1$ and then we obtain the linear recurrence relation

$$
x(s+1)+x(s-1)+\eta x(s)+\zeta=0 .
$$

This equation is well known - it describes the so-called "Askey-Wilson grids" (AW-grid) [1, 14]. The most general is the " $q$-quadratic" grid:

$$
x(s)=c_{1} q^{s}+c_{2} q^{-s}+c_{0}
$$

with some constants $c_{0}, c_{1}, c_{2}$ and a "basic" parameter $q$. In the limit $q=1$ we obtain the quadratic grid:

$$
x(s)=c_{2} s^{2}+c_{1} s+c_{0} .
$$

Further simple analysis of the relation (8.6) allows to obtain explicit expressions for $A(s)$ and $B(s)$. It appears (we omit technical details) that corresponding operator $H$ coincides with the difference Askey-Wilson operator on the grid $x(s)$ (for details see $[1,14,26]$ ). Thus we showed that the only condition (8.6) concerning integrability in the Heisenberg picture of the second-order difference operator leads uniquely to the Askey-Wilson difference operator and corresponding AW-grid. It can be compared with results of [26], where the similar statement was obtained but under different conditions.

It is then directly verified that the second ("dual") algebraic relation

$$
[Y, X]=V_{1}(X) H+V_{2}(X) Y+V_{0}(X)
$$

holds automatically with $\operatorname{deg}\left(V_{2}(X)\right) \leq 1$ and $\operatorname{deg}\left(V_{0,1}(X)\right) \leq 2$.

Combining (8.6) and (8.7) we immediately conclude that operators $H, X$ together with their extension operator $Y=[H, X]$ form the (generic) Askey-Wilson algebra $A W(3)$ (5.2). Thus the $A W(3)$ algebra can be derived uniquely from the "Heisenberg solvability" principle. This results seems to be new. Note that in [15] and [16] it was directly verified that the AskeyWilson Hamiltonian (8.5) (as well as all its special cases corresponding to classical orthogonal polynomials) provides an exact solution for the "grid variable" $X$ in the Heisenberg picture. In our approach these results follow directly from the quasi-linear property of the $A W(3)$ algebra.

Concerning classical (i.e. Poisson brackets) analogue of the $A W(3)$ algebra and corresponding exactly solvable systems see e.g. [29]. 


\section{Conclusion}

We demonstrated that the "quasi-linear" algebras (with possible extension) can be considered as a natural generalization of the Lie algebras: they preserve the linearity property with respect to one-parameter subgroups (exponential mapping) constructed from the fixed set of the "Hamiltonians". This provides an exact time evolution (Heisenberg picture) with respect to all these Hamiltonians. The situation is almost the same in both classical (with Poisson brackets instead of commutators) and quantum picture. Many exactly solvable models in classical and quantum mechanics admit an alternative description in terms of a corresponding quasi-linear algebra.

There are many open questions and problems connected with quasi-linear algebras:

(i) is it possible to give complete classification of all finite-dimensional quasi-linear algebras (with or without extension)? We were able to construct such classification only in the classical case (i.e. Poisson algebras) for the simplest cases of types $(2,0)$ and $(3,0)$ without extensions. We hope that in quantum (operator) case such classification is possible at least in the case when all operators are finite-dimensional.

(ii) in our definition of the quasi-linear algebras, e.g. in condition (2.1) the polynomials $F$ (depending on the Hamiltonians $H$ ) stand to the left side of the operators $X_{k}$. Of course it is possible to define the "right" quasi-linear algebras with the property

$$
\left[H, X_{k}\right]=\sum_{s=1}^{N} X_{s} F_{k s}(H)+\Phi_{k}(H),
$$

instead of (2.1). It is easily verified that for all considered examples of the quasi-linear algebras the "right" version coincides with the "left" version (with possible modification of the structure parameters). Is this property valid in general situation? We hope that the answer is positive.

Note that if the operators $H$ and all $X_{i}$ are Hermitian and if $F_{k s}(x)$ and $\Phi_{k}(x)$ are polynomials in $x$ (as happens e.g. for already considered examples of integrable systems in quantum mechanics) then obviously the "left" and the "right" versions are equivalent; indeed, taking Hermitian conjugation of (9.1) we have

$$
\left[H, X_{k}\right]^{\dagger}=-\left[H, X_{k}\right]=\sum_{s=1}^{N} F_{k s}^{*}(H) X_{s}+\Phi_{k}^{*}(H),
$$

( $F^{*}$ means complex conjugation of corresponding polynomials), i.e. we obtained the "left" version from the initial "right" version. However, from general mathematical point of view we cannot assume that operators $H$ and $X_{i}$ are Hermitian, so the problem of equivalence between the "left" and the "right" version remains open.

(iii) possible applications to exactly solvable models. As we know, the Jacobi algebra $Q J(3)$ describes exactly solvable one-dimensional quantum Hamiltonians with the singular oscillator, Morse and Pöschl-Teller potentials. The Askey-Wilson algebra $A W(3)$ describes all "classical" second-order difference equations. By "classical" we mean that these equations have solutions in terms of classical orthogonal polynomials on non-uniform grids (the most general are the Askey-Wilson difference equations [26]). What about multi-dimensional generalization of these results? An interesting approach was proposed in [3], where the $q$-deformed DG-relations are applied to integrable models like $X X Z$-chain on the one side and to some generalization of the Askey-Wilson polynomials on the other side.

(iv) the "quasi-linear" algebras do not describe all interesting non-linear algebraic objects in theory of integrable systems. Among such algebras are so-called Sklyanin algebras introduced in [21] and then generalized in many papers. The Sklyanin algebra consists from 4 generators $S_{i}$, $i=0, \ldots, 4$ with some special quadratic commutation relations between all possible pairs of the generators. These algebras are closely related with elliptic solutions of the Yang-Baxter equation 
for the 8-vertex model in statistical physics. Recently it was shown that the Sklyanin algebras play an important role in theory of biorthogonal rational functions on elliptic grids [20, 23]. Already on the classical level (Poisson bracket version) the time evolution with respect to any "Hamiltonian" $S_{i}$ is described by elliptic functions [11]. Hence in this case time evolution does not satisfy linearity property with respect to generators. It would be very interesting to find a basic property of these algebras which generalize the quasi-linear property.

\section{Acknowledgments}

A.Zh. thanks Centre de Recherches Mathématiques of the Université de Montréal for hospitality and T. Ito, A. Kiselev, M. Nesterenko and P. Terwilliger for discussions. The authors would like to thank referees for valuable remarks and comments.

\section{References}

[1] Askey R., Wilson J., Some basic hypergeometric orthogonal polynomials that generalize Jacobi polynomials, Mem. Amer. Math. Soc. 54 (1985), no. 319, 1-55.

[2] Baseilhac P., Koizumi K., A deformed analogue of Onsager's symmetry in the XXZ open spin chain, J. Stat. Mech. Theory Exp. 2005 (2005), P10005, hep-th/0507053.

[3] Baseilhac P., Deformed Dolan-Grady relations in quantum integrable models, Nuclear Phys. B 709 (2005), 491-521, hep-th/0404149.

[4] Davies B., Onsager's algebra and superintegrability, J. Phys. A: Math. Gen. 23 (1990), 2245-2261.

[5] Dolan L., Grady M., Conserved charges from self-duality, Phys. Rev. D (3) 25 (1982), 1587-1604.

[6] Fokas A.S., Gelfand I.M., Quadratic Poisson algebras and their infinite-dimensional extensions, J. Math. Phys. 35 (1994), 3117-3131.

[7] Gorsky A.S., Zabrodin A.V., Degenerations of Sklyanin algebra and Askey-Wilson polynomials, J. Phys. A: Math. Gen. 26 (1993), L635-L639, hep-th/9303026.

[8] Granovskii Ya.I., Lutzenko I.M., Zhedanov A.S., Mutual integrability, quadratic algebras, and dynamical symmetry, Ann. Phys. 217 (1992), 1-20.

[9] Ito T., Tanabe K., Terwilliger P., Some algebra related to $P$ - and $Q$-polynomial association schemes, in Codes and Association Schemes (1999, Piscataway NJ), Amer. Math. Soc., Providence RI, 2001, 167-192, math.CO/0406556.

[10] Karasëv M.V., Maslov V.P., Nonlinear Poisson brackets. Geometry and quantization, Translations of Mathematical Monographs, Vol. 119, Amer. Math. Soc., Providence, RI, 1993.

[11] Korovnichenko A., Zhedanov A., Dual algebras with non-linear Poisson brackets, in Integrable Structures of Exactly Solvable Two-Dimensional Models of Quantum Field Theory (2000, Kiev), NATO Sci. Ser. II Math. Phys. Chem., Vol. 35, Kluwer Acad. Publ., Dordrecht, 2001, 265-272.

[12] Korovnichenko A., Zhedanov A., Classical Leonard triples, in Proceedings of Workshop on Elliptic Integrable Systems (November 8-11, 2004, Kyoto), 2004, 71-84, available at http://www.math.kobe-u.ac.jp/publications/rlm18/6.pdf.

[13] Létourneau P., Vinet L., Quadratic algebras in quantum mechanics, in Symmetries in Science, VII (1992, Nakajō), Plenum, New York, 1993, 373-382.

[14] Nikiforov A.F., Suslov S.K., Uvarov V.B., Classical orthogonal polynomials of a discrete variable, Springer, 1991.

[15] Odake S., Sasaki R., Unified theory of annihilation-creation operators for solvable ("discrete") quantum mechanics, J. Math. Phys. 47 (2006), 102102, 33 pages, quant-ph/0605215.

[16] Odake S., Sasaki R., Exact solutions in the Heisenberg picture and annihilation-creation operators, Phys. Lett. B 641 (2006), 112-117, quant-ph/0605221.

[17] Odesskii A., Rubtsov V., Polynomial Poisson algebras with regular structure of symplectic leaves, Teoret. Mat. Fiz. 133 (2002), 1321-1337, math.QA/0110032. 
[18] Perk J.H.H., Star-triangle equations, quantum Lax operators, and higher genus curves, in Proceedings 1987 Summer Research Institute on Theta functions, Proc. Symp. Pure. Math., Vol. 49, Part 1, Amer. Math. Soc., Providence, R.I., 1989, 341-354.

[19] Rosenberg A., Non-commutative algebraic geometry and representations of quantized algebras, Kluwer Academic Publishers, 1995.

[20] Rosengren H., An elementary approach to $6 j$-symbols (classical, quantum, rational, trigonometric, and elliptic), Ramanujan J. 13 (2007), 131-166, math.CA/0312310.

[21] Sklyanin E.K., On some algebraic structures connected with the Yang-Baxter equation, Funct. Anal. Appl. 16 (1982), no. 4, 263-270.

Sklyanin E.K., Some algebraic structures connected with the Yang-Baxter equation. Representations of quantum algebras, Funct. Anal. Appl. 17 (1983), no. 4, 273-284.

[22] Smith S.P., Bell A.D., Some 3-dimensional skew polynomial rings, Preprint, 1991.

[23] Spiridonov V., Zhedanov A., Poisson algebras for some generalized eigenvalue problems, J. Phys. A: Math. Gen. 37 (2004), 10429-10443.

[24] Terwilliger P., Two linear transformations each tridiagonal with respect to an eigenbasis of the other, Linear Algebra Appl. 330 (2001), 149-203.

[25] Terwilliger P., Two relations that generalize the $q$-Serre relations and the Dolan-Grady relations, math.QA/0307016.

[26] Vinet L., Zhedanov A., Generalized Bochner theorem: characterization of the Askey-Wilson polynomials, J. Comput. Appl. Math. 211 (2008), 45-56, arXiv:0712.0069.

[27] Wiegmann P.B., Zabrodin A.V., Algebraization of difference eigenvalue equations related to $U_{q}\left(s l_{2}\right), N u c l e a r$ Phys. B 451 (1995), 699-724, cond-mat/9501129.

[28] Zhedanov A.S., "Hidden symmetry" of Askey-Wilson polynomials, Teoret. Mat. Fiz. 89 (1991), 190-204 (English transl.: Theoret. and Math. Phys. 89 (1991), 1146-1157).

[29] Zhedanov A., Korovnichenko A., 'Leonard pairs' in classical mechanics, J. Phys. A: Math. Gen. 35 (2002), $5767-5780$. 